\title{
The Relationship Between the Impairment of Endothelial Function and Thyroid Antibodies in Hashimoto's Thyroiditis Patients with Euthyroidism
}

Authors

Yanjin Hu*(D, Zhi Yao*, Guang Wang

Affiliation

Department of Endocrinology, Beijing Chao-yang Hospital, Capital Medical University. Beijing P.R. China

Key words

Hashimoto's thyroiditis, endothelial dysfunction, thyroid peroxidase antibody

received $\quad 08.01 .2020$

accepted $\quad 07.05 .2020$

Bibliography

DOI https://doi.org/10.1055/a-1178-5882

Published online: 15.6 .2020

Horm Metab Res 2020; 52: 642-646

(c) Georg Thieme Verlag KG Stuttgart · New York

ISSN 0018-5043

Correspondence

Dr. Guang Wang

Department of Endocrinology, Beijing Chao-yang Hospital

Capital Medical University

Beijing 100020

P. R. China

Tel.: + 861085231710 , Fax: + 861085231710

drwg6688@163.com

\section{ABSTRACT}

Endothelial dysfunction is the important early step in the development of atherosclerosis. Hypothyroidism caused by Hashimoto's thyroiditis and other thyroid disease is one of the risk factors of endothelial dysfunction. The present study tried to investigate the endothelial function and its associated factors in Hashimoto thyroiditis with euthyroidism. A total of 95 newly diagnosed Hashimoto's thyroiditis patients with euthyroidism and 45 healthy controls were studied. Hashimoto's patients were divided into 3 subgroups namely, single thyroglobulin antibody (TGAb) positive subgroup, single thyroid peroxidase antibody (TPOAb) positive subgroup, and both TGAb and TPOAb positive subgroup. Endothelial function was tested by the reactive hyperemia index (RHI). Hashimoto's thyroiditis patients had lower $\mathrm{RHI}$ than healthy controls $(1.73 \pm 0.42$ vs $1.96 \pm 0.51, p<0.05)$. Hashimoto's thyroiditis with single TGAb positive patients had higher $\mathrm{RHI}$ than single TPOAb positive $(1.98 \pm 0.57$ vs. $1.69 \pm 0.33, p<0.05)$ and TGAB + TPOAb positive patients $(1.98 \pm 0.57$ vs. $1.68 \pm 0.42$, $\mathrm{p}<0.05)$. RHI were negatively associated with total cholesterol (TC, $r=-0.215, p<0.05$ ), low density lipoprotein cholesterol (LDL-C, $r=-0.268, p<0.05)$, triglyceride (TG, $r=-0.192$, $p<0.05)$, and TPOAb $(r=-0.288, p<0.05)$. In the regression analysis, LDL- $C(\beta=-0.146, p<0.05)$, TG $(\beta=-0.034, p<0.05)$ and TPOAb $(\beta=-0.001, p<0.05)$ were independently associated with RHI. Hashimoto's patients had poor endothelial function. TPOAb levels were negatively associated with endothelial function.

\section{Introduction}

Atherosclerosis related diseases are the important cause of death in the world along with the changes of life style [1]. The risk factors of atherosclerosis include endothelial dysfunction, diabetes mellitus, dyslipidemia, hypertension, and smoking [2]. Endothelial dysfunction is an important early stage of atherosclerosis and associated with cardiovascular events [3]. The detection of vascular endothelial function is valuable in the prevention of cardiovascular diseases.

Theses authors contributed equally to this research.
Hashimoto's thyroiditis is a common autoimmune thyroid disease in the endocrinology. It is one of the important causes of hypothyroidism [4]. As the cause of deterioration of Hashimoto's thyroiditis, about $50 \%$ of the patients would suffer from hypothyroidism [5]. In the previous researches, overt and subclinical hypothyroidism were the risk factors of endothelial dysfunction and atherosclerosis [6, 7]. But in the Hashimoto's thyroiditis patients with euthyroidism, the research about endothelial function and its associated factors were not enough. The present study tried to investigate the vascular endothelial function in the Hashimoto's thyroiditis with euthyroidism. In addition, we detected the relationship 
between the endothelial function and its associated factors including the thyroid antibodies.

\section{Subjects and Methods}

\section{Subjects}

A total of 95 newly diagnosed Hashimoto's thyroiditis with euthyroidism and 45 sex- and age-matched healthy controls were recruited in the endocrinology department of Beijing Chao-yang hospital from June 2017 to December 2018. Diagnostic criteria for Hashimoto's thyroiditis were: 1 ) The patients had parenchymal heterogeneity according to the neck ultrasound; and 2) Positive for thyroid peroxidase antibody (TPOAb) and/or thyroglobulin antibody (TGAb). The newly diagnosed Hashimoto's patients were recruited according the following standards: 1) Age > 18 years old; 2) Patients should meet the diagnostic criteria for Hashimoto's thyroiditis; and 3) Patients' free T3 (FT3), free T4 (FT4), total T3 (TT3), total T4 (TT4), and thyroid stimulating hormone (TSH) were in the normal range. The healthy controls should have the normal thyroid function and negative TGAb and TPOAb. Subjects were excluded if they had the history of other thyroid disease such as sub-acute thyroiditis, acute suppurative thyroiditis, Graves' disease and thyroid carcinoma, definite atherosclerotic disease such as coronary heart disease and cerebral infarction, hypertension, cute or chronic hepatic and renal diseases, long-term and heavy smoking, taking the drugs that affect vascular endothelial function such as atorvastatin, folic acid, and so on.

Hashimoto's thyroiditis patients were divided into 3 subgroups according their TPOAb and TGAb: 1) TPOAb (+) group: Hashimoto's patients with positive TPOAb and negative TGAb $(n=44) ; 2)$ TGAb (+) group: Hashimoto's patients with positive TGAb and negative TPOAb ( $n=15)$; and 3) TPOAb TGAb (+) group: Hashimoto's patients with positive TPOAb and TGAb $(n=36)$. The reference range of TPOAb: $0-60 \mathrm{IU} / \mathrm{ml}$. The reference range of TGAb: $0-60 \mathrm{IU} /$ $\mathrm{ml}$.

All subjects enrolled in the study gave informed consent. All procedures were conducted in accordance with Declaration of Helsinki. The present research was approved by the Beijing Chao-yang hospital ethics committee.

\section{Laboratory measurements}

All subjects underwent the assessments of the physical examination including height, weight and blood pressure at the fasting state. Blood samples were collected in the morning after $12 \mathrm{~h}$ of fasting including thyroid function (FT3, FT4, TT3, TT4. TSH), thyroid antibodies (TPOAb, TGAb), fasting plasma glucose (FPG), HbA1c, total cholesterol (TC), low-density lipoprotein cholesterol (LDL-C), high-density lipoprotein cholesterol (HDL-C), and triglycerides (TG). Thyroid ultrasound was performed in each subject. Body mass index (BMI) was calculated as $\mathrm{kg} / \mathrm{m}^{2}$.

\section{Endothelial function test}

Endothelial function was tested by EndoPAT 2000 (Itamar Medical, Caesarea, Israel). It was expressed by the reactive hyperemia index $(\mathrm{RHI})$. Reactive hyperemia index was an operator-independent method of endothelial function and associated with many cardiovascular factors $[8,9]$. Patients were asked to keep calm for $12 \mathrm{~h}$. After 30 min of rest, Endo PAT probes were placed on the index fingers of both side of hands to test baseline pulse amplitude for $5 \mathrm{~min}$. Then the blood pressure cuff was inflated to $200-300 \mathrm{mmHg}$ or $60 \mathrm{mmHg}$ above the patients' systolic pressure for $5 \mathrm{~min}$. The ratio of the hyperemic and baseline pulse amplitude was determined after the control arm correction. RHI was measured at fasting state.

\section{Statistical analysis}

Data were analyzed by SPSS 20.0 software (SPSS, Inc., Chicago, IL, USA). Continuous data as age, BMI, TC, LDL-C, HDL-C, FT3, FT4, TT3, TT4, TSH, systolic pressure (SBP), and diastolic pressure (DBP) were expressed as mean $\pm \mathrm{SD}$. Non-normally distributed variables as TG, TPOAb, TGAb were expressed as median (IQR). Continuous data were analyzed by Student's t-test or ANOVA analysis. Non-normally distributed variables were analyzed by nonparametric test. Spearman's rank correlation was used to assess the association between RHI and other parameters. Multiple linear regression was

- Table 1 Baseline characteristics in healthy controls and HT patients.

\begin{tabular}{|c|c|c|}
\hline & HT $n=95$ & Control $n=45$ \\
\hline Age: years & $40.8 \pm 9.0$ & $41.7 \pm 6.9$ \\
\hline Sex: male/female & $42 / 53$ & $13 / 32$ \\
\hline BMI: kg/m² & $24.7 \pm 2.7$ & $24.8 \pm 2.7$ \\
\hline SBP: $\mathrm{mmHg}$ & $122.2 \pm 10.8$ & $118.6 \pm 11.2$ \\
\hline DBP: $\mathrm{mmHg}$ & $78.0 \pm 8.2$ & $75.3 \pm 7.7$ \\
\hline TC: mmol/l & $5.35 \pm 1.09$ & $5.05 \pm 0.94$ \\
\hline LDL-C: mmol/I & $3.08 \pm 0.83$ & $2.84 \pm 0.66$ \\
\hline HDL-C: mmol// & $1.16 \pm 0.28$ & $1.19 \pm 0.20$ \\
\hline TG: mmol/l & $1.83(1.27,2.77)$ & $1.32(1.02,2.83)$ \\
\hline FPG: mmol/l & $5.23 \pm 0.56$ & $5.22 \pm 0.62$ \\
\hline HbA1c: mmol/mol & $39.30 \pm 4.20$ & $37.44 \pm 10.43$ \\
\hline TT3: ng/ml & $1.21 \pm 0.36$ & $1.19 \pm 0.35$ \\
\hline TT4: $\mu \mathrm{g} / \mathrm{dl}$ & $7.55 \pm 2.17$ & $7.82 \pm 1.94$ \\
\hline FT3: pg/ml & $3.24 \pm 0.71$ & $3.01 \pm 0.73$ \\
\hline FT4: ng/dl & $1.29 \pm 0.31$ & $1.30 \pm 0.37$ \\
\hline TSH: $\mu \mathrm{lU} / \mathrm{ml}$ & $2.65 \pm 1.16$ & $2.52 \pm 1.10$ \\
\hline TPOAB: IU/ml & $\begin{array}{l}112.3(71.8 \\
195.5)^{*}\end{array}$ & $28.0(13.2,33.0)$ \\
\hline TGAB: IU/ml & $75.4(23.1,233.3)^{*}$ & $16.2(6.2,27.2)$ \\
\hline RHI & $1.73 \pm 0.42^{*}$ & $1.96 \pm 0.51$ \\
\hline \multicolumn{3}{|c|}{$\begin{array}{l}\text { Data are expressed as means } \pm \text { SD or medians (inter-quartile ranges). } \\
\text { HT: Hashimoto’s thyroiditis; BMI: Body mass index; SBP: Systolic } \\
\text { pressure; DBP: Diastolic pressure; TC: Total cholesterol; LDL-C: } \\
\text { Low-density lipoprotein cholesterol; HDL-C: High-density lipoprotein } \\
\text { cholesterol; TG: Triglycerides; TT3: Total T3; TT4: Total t4; FT3: Free } \\
\text { T3; FT4: Free T4; TSH: Thyroid stimulating hormone; RHI: Reactive } \\
\text { hyperemia index. }{ }^{*} \mathrm{p}<0.05 \text { vs. } \pm \text { Control group. }\end{array}$} \\
\hline
\end{tabular}


used to assess the associated factors of RHI. All analyses were two tailed and $p<0.05$ were considered statistically significant.

\section{Results}

\section{Baseline characteristics of Hashimoto's thyroiditis patients and control group}

The baseline characteristics of subjects are shown in $>$ Table 1. The blood pressure, lipid metabolism, FPG, HbA1c, age, sex, and thyroid function were similar between Hashimoto's thyroiditis and control group. Hashimoto's thyroiditis patients had lower RHI $(1.73 \pm 0.42$ vs. $1.96 \pm 0.51, \mathrm{p}<0.05)$ and higher TPOAb [112.3 $(71.8,195.5)$ vs. $28.0(13.2,33.0) \mathrm{IU} / \mathrm{ml}, \mathrm{p}<0.05]$ and TGAb [75.4 $(23.1,233.3)$ vs. $16.2(6.2,27.2) \mathrm{IU} / \mathrm{ml}, \mathrm{p}<0.05]$ than control groups.

\section{Subgroup analysis of Hashimoto's thyroiditis patients and control group}

To detect the relationship between endothelial dysfunction and thyroid antibodies, we did the subgroup analyses according the TGAb and TPOAb titer. In the sub-group analysis, age, sex, blood pressure, TC, TG, LDL-C, HDL-C, FPG, HbA1c, and thyroid function were similar in the 3 subgroup and control group. TPOAb (+) and
TPOAb + TGAb ( + ) subgroups had lower RHI than TGAb (+ ) subgroup and control group ( $\triangleright$ Table 2 ).

\section{Correlation between $\mathrm{RHI}$ and other parameters}

The correlation analyses were conducted to test the associations between vascular endothelial dysfunction and other parameters ( $\triangleright$ Table 3). RHI were negatively related to the LDL-C $(r=-0.268$, $p<0.05)$, TG $(r=-0.192, p<0.05)$, and TPOAb $(r=-0.288$, $\mathrm{p}<0.01)$.

\section{Multiple regressions about $\mathrm{RHI}$ and its related factors}

- Table 4 showed the results of multiple regressions of various independent variables to test their association with RHI. LDL-C, TG, and TPOAb were entered in the regression model. Multiple regression analysis demonstrated that LDL-C $(\beta=-0.146, p<0.05)$, TG $(\beta=-0.034, p<0.05)$, and TPOAb $(\beta=-0.001, p<0.05)$ were independently associated with RHI.

\section{Discussion}

The current study demonstrates that Hashimoto's disease had poorer endothelial function than healthy controls and TPOAb was independently associated with endothelial function. In addition,

- Table 2 Comparison of clinical parameters of subgroups of Hashimoto patients and controls

\begin{tabular}{|c|c|c|c|c|}
\hline \multirow[t]{2}{*}{ Parameters } & Control & TPOAb (+) & TGAb (+) & TPOAb + TGAb (+) \\
\hline & $n=45$ & $n=44$ & $n=15$ & $n=36$ \\
\hline Age, years & $41.7 \pm 6.9$ & $40.8 \pm 8.2$ & $44.3 \pm 11.9$ & $39.3 \pm 8.6$ \\
\hline BMI, kg/m² & $24.8 \pm 2.7$ & $24.1 \pm 2.3$ & $24.2 \pm 3.3$ & $25.6 \pm 2.7$ \\
\hline SBP, mmHg & $118.6 \pm 11.2$ & $120.6 \pm 10.4$ & $125.1 \pm 9.9$ & $122.9 \pm 11.5$ \\
\hline DBP, $\mathrm{mmHg}$ & $75.3 \pm 7.7$ & $77.8 \pm 6.8$ & $81.5 \pm 7.7$ & $76.7 \pm 9.7$ \\
\hline $\mathrm{TC}, \mathrm{mmol} / \mathrm{I}$ & $5.05 \pm 0.94$ & $5.43 \pm 1.09$ & $4.92 \pm 1.06$ & $5.44 \pm 1.10$ \\
\hline LDL-C, mmol/I & $2.84 \pm 0.66$ & $3.05 \pm 0.88$ & $2.86 \pm 0.77$ & $3.21 \pm 0.80$ \\
\hline HDL-C, mmol/l & $1.19 \pm 0.20$ & $1.18 \pm 0.32$ & $1.18 \pm 0.19$ & $1.13 \pm 0.26$ \\
\hline $\mathrm{TG}, \mathrm{mmol} / \mathrm{l}$ & $1.32(1.02,2.83)$ & $1.99(1.32,2.98)$ & $1.73(1.05,4.91)$ & $1.78(1.26,2.46)$ \\
\hline $\mathrm{FPG}, \mathrm{mmol} / \mathrm{l}$ & $5.22 \pm 0.62$ & $5.32 \pm 0.52$ & $4.97 \pm 0.49$ & $5.23 \pm 0.60$ \\
\hline $\mathrm{HbA} 1 \mathrm{c}, \mathrm{mmol} / \mathrm{mol}$ & $37.44 \pm 10.43$ & $40.66 \pm 3.90$ & $38.58 \pm 4.39$ & $37.95 \pm 4.06$ \\
\hline TT3, ng/ml & $1.19 \pm 0.35$ & $1.19 \pm 0.32$ & $1.09 \pm 0.35$ & $1.29 \pm 0.40$ \\
\hline TT4, $\mu \mathrm{g} / \mathrm{dl}$ & $7.82 \pm 1.94$ & $7.42 \pm 2.27$ & $7.57 \pm 2.35$ & $7.70 \pm 2.03$ \\
\hline $\mathrm{FT} 3, \mathrm{pg} / \mathrm{ml}$ & $3.01 \pm 0.73$ & $3.32 \pm 0.65$ & $3.05 \pm 0.73$ & $3.23 \pm 0.77$ \\
\hline FT4, ng/dl & $1.30 \pm 0.37$ & $1.27 \pm 0.27$ & $1.25 \pm 0.27$ & $1.35 \pm 0.36$ \\
\hline TSH, $\mu \mathrm{lU} / \mathrm{ml}$ & $2.52 \pm 1.10$ & $2.50 \pm 1.12$ & $2.36 \pm 1.36$ & $2.97 \pm 1.09$ \\
\hline TPOAb, IU/ml & $28.0(13.2,33.0)$ & $136.6(90.5,199.2)$ & $33.0(12.0,50.1)$ & $135.6(91.9,205.8)$ \\
\hline TGAb, IU/ml & $16.2(6.2,27.2)$ & $22.8(2.4,34.2)$ & $238.4(123.4,636.8)$ & $166.0(103.2,331.6)$ \\
\hline $\mathrm{RHI}$ & $1.96 \pm 0.51$ & $1.69 \pm 0.33^{a, b}$ & $1.98 \pm 0.57$ & $1.68 \pm 0.42^{a, b}$ \\
\hline
\end{tabular}

BMI: Body mass index; SBP: Systolic pressure; DBP: Diastolic pressure; TC: Total cholesterol; LDL-C: Low-density lipoprotein cholesterol; HDL-C: High-density lipoprotein cholesterol; TG: Triglycerides; TT3: Total T3; TT4: Total t4; FT3: Free T3; FT4: Free T4; TSH: Thyroid stimulating hormone; RHI: Reactive hyperemia index. $\mathrm{p}<0.05$ vs. Control group. ${ }^{\mathrm{b}} \mathrm{p}<0.05$ vs. TGAb $(+)$ group. 
- Table 3 Correlation analyses of the parameters associated with RHI.

\begin{tabular}{|l|c|c|}
\hline Parameters & $\mathbf{r}$ & p-Value \\
\hline Age, years & 0.037 & 0.662 \\
\hline BMI, kg/m² & 0.055 & 0.519 \\
\hline SBP, mmHg & -0.041 & 0.629 \\
\hline DBP, mmHg & -0.057 & 0.500 \\
\hline TC, mmol/l & -0.215 & 0.011 \\
\hline LDL-C, mmol/I & -0.268 & 0.001 \\
\hline HDL-C, mmol/l & 0.032 & 0.704 \\
\hline TG, mmol/l & -0.192 & 0.023 \\
\hline FPG, mmol/l & -0.056 & 0.508 \\
\hline HbA1C, mmol/mol & -0.102 & 0.232 \\
\hline TT3, ng/ml & -0.058 & 0.494 \\
\hline TT4, $\mu$ g/dl & 0.057 & 0.506 \\
\hline FT3, pg/ml & -0.116 & 0.174 \\
\hline FT4, ng/dl & 0.010 & 0.906 \\
\hline TSH, $\mu$ IU/ml & 0.015 & 0.858 \\
\hline TPOAb, IU/ml & -0.288 & 0.001 \\
\hline TGAb, IU/ml & -0.053 & 0.537 \\
\hline BMI: Body mass index; SBP: Systolic pressure; DBP: Diastolic \\
\hline pressure; TC: Total cholesterol; LDL-C: Low-density lipoprotein \\
\hline cholesterol; HDL-C: High-density lipoprotein cholesterol; TG: \\
\hline Triglycerides; TT3: Total T3; TT4: Total t4; T3: Free T3; FT4: Free T4; \\
\hline TSH: Thyroid stimulating hormone; RHI: Reactive hyperemia index. \\
\hline
\end{tabular}

- Table 4 Multiple stepwise regression analysis of the parameters associated with RHI.

\begin{tabular}{|l|l|l|l|}
\hline Parameters & $\boldsymbol{\beta}$ & SE & p-Value \\
\hline LDL-C, mmol/l & -0.146 & 0.047 & 0.002 \\
\hline TG, mmol/l & -0.034 & 0.014 & 0.020 \\
\hline TPOAb, IU/ml & -0.001 & 0.000 & 0.003 \\
\hline
\end{tabular}

LDL-C: Low-density lipoprotein cholesterol; TG: Triglycerides; RHI: Reactive hyperemia index.

we found that Hashimoto's patients with TPOAb positive or both TPOAb and TGAb positive had worse endothelial dysfunction than the single TGAb positive group.

In the previous study, it was noted that overt hypothyroidism and subclinical hypothyroidism could lead to the endothelial dysfunction [10]. The mechanisms include promoting oxidative stress, aggravating insulin resistance, elevating serum homocysteine, and so on [11-13]. Hashimoto's thyroiditis is the most important cause of hypothyroidism. But in the Hashimoto's thyroiditis with euthyroidism, the number of research about endothelial dysfunction is not much. In our study, Hashimoto's thyroiditis patients had the similar age, sex, BMI, blood pressure, and lipid level with the control, but had lower RHI level. This is consistent with the previous studies. Xiang et al. found the Hashimoto's thyroiditis had poor endothelial function but the sample size was not large $(n=28)$ [14]. In addition, another research with larger samples in adolescent Hashimoto's thyroiditis found that they had significantly increased carotid intima-media thickness (cIMT) [15]. cIMT is a good tool for the early detection of early atherosclerosis. The reasons for this phenomenon are not yet clear. It is generally believed that this may be related to the chronic inflammation in the Hashimoto's thyroiditis patients. In the previous studies, Hashimoto's patients had increased $C$ reactive protein, interleukin 6 , interleukin $1 \beta$, and tumor necrosis factor $\alpha$ levels. It is suggested that Hashimoto's patients were in the state of chronic inflammation $[16,17]$. In addition, Hashimoto's patients also had higher levels of soluble intercellular adhesion molecule 1 (sICAM-1) and soluble vascular cell adhesion molecule-1 (sVCAM-1) although they were in the euthyroidism states [18]. Many researches demonstrated that chronic inflammation cause endothelial dysfunction in the human umbilical vein endothelial cells $[19,20]$. With the state of chronic inflammation and the progressive decrease of thyroid function, Hashimoto's thyroiditis patients become a risk group of atherosclerosis and cardiovascular and cerebrovascular disease.

In the next step, we found that Hashimoto's thyroiditis patients with positive TPOAb whether TGAb levels were positive or not had poorer endothelial function than patients with single positive TGAb levels. TPOAb levels were independent negatively associated with the endothelial function but not TGAb. These suggested that TPOAb may be more closely related to the vascular endothelial dysfunction. TPOAb is produced by lymphocyte and presents in nearly every Hashimoto's thyroiditis patients [21]. It is also the most sensitive marker for Hashimoto's thyroiditis and associate with the degree of infiltration by lymphocytes. TPOAb is able to induce and activate the complement system and exert the cellular cytotoxicity [17]. In the previous studies, TPOAb levels were positively related to the $C$ reactive protein and multiple pro-inflammatory cytokines such as TNF- $\alpha$, interleukin-6, and interferon- $\gamma$ [22]. . The chronic inflammation may due to the positive association between the TPOAb and endothelial dysfunction [14].

In contrast, we did not find the endothelial dysfunction in the Hashimoto's thyroiditis patients with single TGAb positive and the relationship between TGAb and endothelial function in Hashimoto's thyroiditis. In the previous studies, the relationships between TGAb and endothelial dysfunction were various $[14,16]$. The possible reason is that TGAb could not activated complement system and cellular cytotoxicity [23]. The inflammatory effect of TGAb may be less than TPOAb. In addition, the high detection rate of TPOAb in Hashimoto's thyroiditis caused the sample size of single TCAB positive group was not large. This may lead to the bias of results.

In the multiple regression, we found that the LDL-C and TG were negatively associated with RHI. It was consistent with previous studies. In our research about the endothelial function in type 2 diabetes, LDL-C is the risk factors of endothelial dysfunction [24]. LDL-C increases oxidative stress and impaired the endothelial nitric oxide synthase and reduces the nitric oxide production [25]. In the overt or subclinical hypothyroidism patients, the elevated LDL-C is a risk 
factor of atherosclerosis. The relationship between TG and endothelial dysfunction and cardiovascular disease were investigated in many researches $[26,27]$. The mechanism included the increased of triglyceride-rich lipoproteins (TRL). TRL remnants are small enough to enter the arterial wall and promote inflammation and foam cell generation [28]. It leads to the endothelial dysfunction and atherosclerosis.

Some limitations of our research should be mentioned. First, we did not test the inflammatory marker, especially the factors related to the endothelial dysfunction. Second, Hashimoto's patients with single TGAb positive group's sample size were small. Finally, the diagnosis of Hashimoto's thyroiditis relied on the ultrasound and antithyroid antibodies. Pathological diagnosis might make the results more convincing.

\section{Conclusion}

Endothelial dysfunction exists in the Hashimoto's thyroiditis patients. Patients with positive TPOAb had poorer endothelial function. TPOAb levels were independently negatively associated with the severity of endothelial dysfunction.

\section{Conflict of Interest}

The authors declare that they have no conflict of interest.

\section{References}

[1] Roth GA, Forouzanfar MH, Moran AE et al. Demographic and epidemiologic drivers of global cardiovascular mortality. N Engl J Med 2015; 372: 1333-1341

[2] Lusis A]. Atherosclerosis. Nature 2000; 407: 233-241

[3] Gimbrone MA Jr., García-Cardeña G. Endothelial cell dysfunction and the pathobiology of atherosclerosis. Circ Res 2016; 118: 620-636

[4] Antonelli A, Ferrari SM, Corrado A et al. Autoimmune thyroid disorders. Autoimmun Rev 2015; 14: 174-180

[5] Huber G, Staub J], Meier C et al. Prospective study of the spontaneous course of subclinical hypothyroidism: Prognostic value of thyrotropin, thyroid reserve, and thyroid antibodies. J Clin Endocrinol Metab 2002; 87: 3221-3226

[6] Hak AE, Pols HA, Visser T] et al. Subclinical hypothyroidism is an independent risk factor for atherosclerosis and myocardial infarction in elderly women: The Rotterdam Study. Ann Intern Med 2000; 132: 270-278

[7] Saif A, Mousa S, Assem M et al. Endothelial dysfunction and the risk of atherosclerosis in overt and subclinical hypothyroidism. Endocr Connect 2018; 7: 1075-1080

[8] Hamburg NM, Keyes M], Larson MG et al. Cross-sectional relations of digital vascular function to cardiovascular risk factors in the Framingham Heart Study. Circulation 2008; 117: 2467-2474

[9] Koska J, Schwartz EA, Mullin MP et al. Improvement of postprandial endothelial function after a single dose of exenatide in individuals with impaired glucose tolerance and recent-onset type 2 diabetes. Diabetes Care 2010; 33: 1028-1030
[10] Lu M, Yang CB, Gao L et al. Mechanism of subclinical hypothyroidism accelerating endothelial dysfunction (Review). Exp Ther Med 2015; 9: $3-10$

[11] Dong X, Yao Z, Hu Y et al. Potential harmful correlation between homocysteine and low-density lipoprotein cholesterol in patients with hypothyroidism. Medicine (Baltimore) 2016; 95: e4291

[12] Yang N, Yao Z, Miao L et al. Novel clinical evidence of an association between homocysteine and insulin resistance in patients with hypothyroidism or subclinical hypothyroidism. PLoS One 2015; 10: e0125922

[13] Chakrabarti SK, Ghosh S, Banerjee S et al. Oxidative stress in hypothyroid patients and the role of antioxidant supplementation. Indian J Endocrinol Metab 2016; 20: 674-678

[14] Xiang GD, He YS, Zhao LS et al. Impairment of endothelium-dependent arterial dilation in Hashimoto's thyroiditis patients with euthyroidism. Clin Endocrinol (Oxf) 2006; 64: 698-702

[15] İşgüven P, Gündüz Y, Kılıç M. Effects of thyroid autoimmunity on early atherosclerosis in euthyroid girls with hashimoto's thyroiditis. J Clin Res Pediatr Endocrinol 2016; 8: 150-156

[16] Mikoś H, Mikoś M, Obara-Moszyńska $M$ et al. The role of the immune system and cytokines involved in the pathogenesis of autoimmune thyroid disease (AITD). Endokrynol Pol 2014; 65: 150-155

[17] Liu J, Duan Y, Fu J et al. Association between thyroid hormones, thyroid antibodies, and cardiometabolic factors in non-obese individuals with normal thyroid function. Front Endocrinol (Lausanne) 2018; 9: 130

[18] Ozderya A, Aydin K, Temizkan S et al. High circulating levels of sICAM-1 and SVCAM- 1 in the patients with Hashimoto's thyroiditis. Endocr Res 2017; 42: 110-116

[19] Da Silva MS, Bilodeau JF, Larose J et al. Modulation of the biomarkers of inflammation and oxidative stress by ruminant trans fatty acids and dairy proteins in vascular endothelial cells (HUVEC). Prostaglandins Leukot Essent Fatty Acids 2017; 126: 64-71

[20] Mohr T, Haudek-Prinz V, Slany A et al. Proteome profiling in IL-1 $\beta$ and VEGF-activated human umbilical vein endothelial cells delineates the interlink between inflammation and angiogenesis. PLoS One 2017; 12: e0179065

[21] Czarnocka B, Janota-Bzowski M, McIntosh RS et al. Immunoglobulin G kappa antithyroid peroxidase antibodies in Hashimoto's thyroiditis: epitope-mapping analysis. J Clin Endocrinol Metab 1997; 82: 2639-2644

[22] Nielsen CH, Brix TH, Leslie RG et al. A role for autoantibodies in enhancement of pro-inflammatory cytokine responses to a self-antigen, thyroid peroxidase. Clin Immunol 2009; 133: 218-227

[23] Fink H, Hintze G. Autoimmune thyroiditis (Hashimoto's thyroiditis): current diagnostics and therapy. Med Klin (Munich) 2010; 105: 485-493

[24] Yu J, Han JL, He LY et al. Low density lipoprotein cholesterol level inversely correlated with coronary flow velocity reserve in patients with Type 2 diabetes. J Geriatr Cardiol 2013; 10: 159-164

[25] Hermida N, Balligand JL. Low-density lipoprotein-cholesterol-induced endothelial dysfunction and oxidative stress: The role of statins. Antioxid Redox Signal 2014; 20: 1216-1237

[26] Kajikawa M, Maruhashi T, Matsumoto T et al. Relationship between serum triglyceride levels and endothelial function in a large community-based study. Atherosclerosis 2016; 249: 70-75

[27] Reiner ž. Are elevated serum triglycerides really a risk factor of coronary artery disease? Cardiology 2015; 131: 225-227

[28] Schwartz EA, Reaven PD. Lipolysis of triglyceride-rich lipoproteins, vascular inflammation, and atherosclerosis. Biochim Biophys Acta 2012; 1821: 858-866 